\title{
The needs for care assessment - a longitudinal approach
}

\author{
Denis O'Leary and Marcus Webb
}

\begin{abstract}
This report describes the use of the needs for care assessment (NFCA) in measuring symptom and social function in patients with long-term mental illness. The schedule recommends specilic core interventions and these were implemented where approprlate. The problem status of the patients was reassessed using the needs for care schedule ofier the interventions had been offered and tried. The recommended care thems were within the compass of our rehablilitation service, were rarely refused by the patients and there was an improvement in symplom and social tunction. These findings provide some valldation for the concept of unmet noed.
\end{abstract}

In concept and in use the needs for care assessment (NFCA) aims to set up minimum standards of care for patients in regular contact with psychiatric services (Brewin \& Wing, 1989). Problems in 20 clinical and social areas are assessed by patient or relative/staff interview. The instrument lists interventions ('items of care') for redressing these problems. A staff member is then interviewed to assess whether these items of care would be appropriate, or are in place. The need status 'unmet need' signals problems where minimum standards of care are lacking, i.e. the appropriate care item has not been recently or adequately tried.

The care items are 'ideal' in that they are rated independently of avallability of care and of the care philosophy adopted in a given setting. However, they are defined by experts, and their validity (and the validity of the concept of unmet need) can be assessed only by offering them to patients, implementing them and assessing outcome (Brewin \& Wing, 1993). It would be illogical to suggest that minimum standards of care were not being met if the 'expert' defined care items proposed by the schedule were unlikely to improve functioning levels.

Clearly longitudinal studies are needed. While the NFCA has been used cross-sectionally (Brugha et al, 1988; Lesage et al, 1991; Hogg \& Marshall, 1992: Pryce et al. 1991, 1993) we are not aware of longitudinal studies. In this paper we report how a multidisciplinary team used the NFCA longitudinally, and comment on avallability, acceptability and effectiveness of the care items proposed by the NFCA.

\section{The study}

\section{Patients}

Eighteen patients referred to a high support hostel from a Dublin district psychiatric sector (population 89000 ) were assessed. Their median age was age 43 (range 21-64 years). Fourteen met DSM-III-R criteria for schizophrenia and four had affective disorders. The sample included ten males. The median length of psychiatric illness was $17(2-40)$ years. Nine were in-patients (due to lack of adequate hostel facilities). The remaining nine resided at home with regular service contact.

\section{Assessment and assessors}

Three pairs of staff assessed six patients each using the NFCA and the Social Behaviour Schedule (SBS; Wykes \& Sturt, 1986). To facilitate repeat assessments using the NFCA, we designed a semi-structured interview schedule. Another pair rated all patients using the Present State Examination (PSE; Wing et al, 1974).

Level of functioning in 20 social and clinical areas was assessed by questioning the patient or relatives/staff in close contact with them. The NFCA rates problems as 'current' if the level of function fell below a standard threshold within the previous month. If the level of function is above the threshold required for the previous month, yet has fallen below it in the previous two years, or is likely to in the future, the problem is rated as 'recent'. Staff were then interviewed to assess the items of care in place for dealing with these problems.

Primary need status was rated as unmet when a problem was identified and when the care item was considered appropriate, yet had not been given a recent and/or adequate trial.

Following assessment, the clinical teams responsible for each patient were notified of the results. Items of care were offered and provided on a one to one level by the clinical teams or where appropriate, in groups. 
Needs status were re-assessed 3-6 months after the initial assessment by the same pairs of workers interviewing the same informants as before. To avoid bias, interviewers did not assess patients from their own clinical team and informants were selected as far as possible who had minimal direct involvement in implementing the items of care. Statistical analysis was by Wilcoxon matched-pairs signed-ranks test.

\section{Findings}

\section{Initial assessment}

On first assessment, 109 current and 29 recent problems were identified from a possible 360 (18 patients $\times 20$ functional areas). The range of current and recent problems per patient was 1-15 (median=6) and 0-5 (median=1.0) respectively. For one patient who died prior to the second assessment, details (4 current/ 1 recent problem) were excluded from further analysis.

A total of 51 unmet needs for treatment were identified $(51 / 133=38 \%)$. The related problems in the clinical area included psychotic symptoms (3), underactivity (3), physical illness (3), embarrassing behaviour (4), psychosocial distress (7) and other (3). In the social area, problems were with personal hygiene (4), household shopping (4), occupation (4), communication skills (5), money management (7) and other (4). More than half of the care items recommended in the clinical areas related to suppport/reassurance or coping advice to the subject $(13 / 23=57 \%)$. In the social domain. 28 care items were recommended, with remedial training for personal hygiene, household shopping and money management being the most common. All these care items were within the capacity of the service to provide. Of the remaining problems, 52 were rated as met need, six as unmet need for assessment, and 24 as no meetable need.

\section{Repeat assessment}

The problem, item of care and resultant needs status after 3-6 months are presented in Table 1 . By this time one patient had been discharged from in-patient care and four out-patients had been placed in group homes.

Thirty-three items of care were actually offered to patients. One patient refused the offered care item. In two instances the care items were judged as unsuccessful in improving functional level. In 17 instances $(51 \%)$ the care items care were judged successful, including a subgroup of six in which the intervention was continued to prevent recurrence. In a further 13 instances $(40 \%)$, although functional level did improve, but not to the required threshold, the items were considered appropriate and worthy of continuation.
Table 1. Level of function, item of care and need status at the second assessment

\begin{tabular}{|c|c|c|c|}
\hline LOF & IOC status & No. of IOC status & Need stotus \\
\hline 1 & 0 & 11 & no meetable need \\
\hline 1 & 1 & 6 & met \\
\hline 2 & 2 & 13 & met \\
\hline 2 & 3 & 1 & no meetable need \\
\hline 2 & 5 & 11 & no meetable need \\
\hline 2 & 7 & 9 & unmet \\
\hline
\end{tabular}

LOF, level of function (1=recent, $2=$ current)

$1 O C$. item of care $(0=1$ tem of care not appropriate and not provided; $l=$ currently provided and effective or potentially effective; $2=c$ urrently being provided but had proved to be insufficient in itself after 3 months trial. The form of care is still appropriate and worth continuing: $3=i t e m$ of care offered during the past year but refusal, premature termination or nonattendance by the patient: 5=appropriate, but not provided due to incapacitating symptoms or danger of overload; 7=appropriate now but not given adequate or recent trial)

Eighteen items of care were not offered. These included eleven instances where intervention was judged in danger of overloading two patients suffering schizophrenic relapses. Four were not implemented as the patient involved had left the catchment area and three were overlooked (all seven yielded unmet need status at follow-up). The reduction in the median number of unmet needs per patient was significant $(P<0.001)$.

\section{Comment}

Both in conception and use the NFCA aims to set minimum standards of care. These minimum standards are defined by experts (normative) as are the care items proposed as necessary in maintaining such a minimum standard. Although the care items are listed whether or not the service can provide them, we found that all those required in the present report were within the compass of our service. This finding. together with the fact that only one care item was refused by a patient, indicate that the care items were at least above the minimum threshold of acceptability to our service and to the patients it served.

Inherent in the concept of unmet need status is that sub-standard levels of function are remediable by listed care items. Given the thresholds for level of function as proposed by the NFCA we found support for this contention. Thirty-three care items were offered and of these $17(51 \%)$ resulted in improvement above the threshold level. In a further $13(40 \%)$, although the improvement was not sufficient to break the 
threshold level of functioning, the care items were considered worthy of continuation. Therefore, some improvement occurred with $91 \%$ of the care items. Indeed, factors limiting improvement were those preventing adequate trial of the care items such as acute relapse or moving out of the catchment area.

The clinical improvement resulted in five patients achieving levels of function higher than those required for high support hostel placement. Such improvement underscores the NFCA philosophy of rating level of function with reference to independent community living.

However, the improvement was rated qualitatively and by staff-based informants. While the improvement provides support for the validity of the care items proposed in the NFCA, more complete validation requires replication by other centres, using independent and quantitative assessments.

In conclusion, we found that the NFCA could be used by a multidisciplinary team longitudinally. We found evidence to support the concept of unmet need in that the care items were rarely refused by the patients and resulted in some improvement in level of function in $91 \%$ of instances. We hope that these findings will encourage further validating research and the wider use of the NFCA.

\section{Acknowledgements}

The authors would like to thank F. Costello, R. Gribbin, E. McCarthy, G. O'Brien, T. O'Brien and $\mathrm{S}$. Toone.

\section{References}

BREWIN, C. R. \& WiNG. J. K. (1989) Manual of the MRC Needs for Care Assessment. MRC Social Psychiatry Unit. London: Institute of Psychiatry.
- \& - (1993) The MRC Needs for Care Assessment progress and controversies. Psychological Medicine. 23. 837-841.

BRUGHA, T. S., Wing, J. K. BREWIN, C. R., et al (1988) The problems of people in long-term psychiatric care. Psychological Medicine, 18, 443-456.

HOGG, L. I. \& MARSHALL, M. (1992) Can we measure need in the homeless mentally ill? Usuing the MRC needs for care assessment in hostels for the homeless. Psychological Medicine, 22, 1027-1034.

lesage, A. D., Mignol. G.. Faccincani, C., et al (1991) Standardised assessment of the needs for care in a cohort of patients with schizophrenic psychoses. In Community Based Psychiatry: Long-Term Patterns of Care in South Verona. Psychological Medicine Monograph Supplement 19. Cambridge: Cambridge Untversity Press.

PRYCE. L. G.. GRIFrTtHS, R. D., GENTRY, R. M., et al (1991) The nature and severity of disabilities in long-stay psychiatric in-patients in South Glamorgan. British Joumal of Psychiatry, 158, 817-821.

- . - - - et al (1993) How important is the assessment of social skills in current long-stay in-patients? British Journal of Psychiatry. 162, 498-502.

Wing. J. K. COOPER. J. E. \& SARTORIUS, N. (1974) Measurement and Classification of Psychiatric Symptoms: an Instruction Manual of the PSE and Catego Program. Cambridge: Cambridge Untversity Press.

WYKES, T. \& STURT, E. (1986) The measurement of soctal behaviour in psychiatric patients: an assessment of the reliability and validity of the SBS schedule. Brttish Joumal of Psychiatry, 148, 1-11.

*Denis O'Leary, Clinical Lecturer, Department of Psychiatry, Addenbrooke's Hospital, Hills Road, Cambridge CB2 2Q9: and Marcus Webb, Professor of Psychiatry. Department of Psychiatry. University of Dublin. Trinity College. St. James' Hospital, Dublin 8, Ireland

*Correspondence 